La Revue

des Droits

de l'Homme

\section{La Revue des droits de l'homme}

Revue du Centre de recherches et d'études sur les droits fondamentaux

Actualités Droits-Libertés | 2018

\title{
Le prix du genre
}

Note sous CEDH, Carvalho Pinto De Sousa Morais c. Portugal (Art. 8 et 14)

\section{Lisa Carayon et Julie Mattiussi}

\section{(2) OpenEdition}

\section{Journals}

Édition électronique

URL : http://journals.openedition.org/revdh/3787

DOI : $10.4000 /$ revdh.3787

ISSN : 2264-179X

\section{Éditeur}

Centre de recherches et d'études sur les droits fondamentaux

\section{Référence électronique}

Lisa Carayon et Julie Mattiussi, «Le prix du genre », La Revue des droits de I'homme [En ligne], Actualités Droits-Libertés, mis en ligne le 12 mars 2018, consulté le 19 avril 2019. URL : http:// journals.openedition.org/revdh/3787 ; DOI : 10.4000/revdh.3787

Ce document a été généré automatiquement le 19 avril 2019

Tous droits réservés 


\title{
Le prix du genre
}

Note sous CEDH, Carvalho Pinto De Sousa Morais c. Portugal (Art. 8 et 14)

\author{
Lisa Carayon et Julie Mattiussi
}

1 En l'espèce, la demanderesse était une femme portugaise née en 1945. En 1993, une maladie gynécologique lui a été diagnostiquée alors qu'elle était âgée de quarante-huit ans. Après plusieurs traitements, une chirurgie a été pratiquée en 1995. Quelques temps plus tard, la patiente a commencé à ressentir d'intenses douleurs, en particulier lorsqu'elle était assise ou en train de marcher. Elle souffrait également d'une perte de sensation au niveau du vagin, ainsi que d'incontinence urinaire. Il est alors apparu que le nerf innervant le périnée avait été endommagé pendant l'opération. La patiente a donc introduit une action en justice à l'encontre de l'établissement hospitalier pour obtenir l'indemnisation de son dommage corporel le 26 avril 2000. Devant la juridiction de première instance, elle demandait, d'une part, à ce que son préjudice matériel soit réparé à hauteur de 75000 euros environ et, d'autre part, à ce que son préjudice moral soit indemnisé par la somme de 250000 euros. Les juges, ayant caractérisé l'existence d'une faute de l'établissement hospitalier, ont condamné ce dernier à indemniser la patiente à hauteur de 92000 euros au titre du préjudice matériel et de 80000 euros au titre du préjudice moral.

2 Un appel a été interjeté par l'établissement hospitalier, tandis qu'une demande reconventionnelle a été formulée par la requérante, qui demandait une revalorisation de son indemnisation. La juridiction d'appel a rendu une décision le 9 octobre 2014 dans laquelle elle n'a pas remis en cause le principe de la responsabilité de l'établissement hospitalier, mais a abaissé la somme octroyée au titre du préjudice moral de 80000 à 50000 euros. La décision était motivée par plusieurs arguments, mais celui sur lequel se concentre la CEDH, et qui fera l'objet du présent commentaire, est soulevé en dernier, à titre additionnel, par la juridiction d'appel portugaise. Il s'agit du motif suivant :

3 «En outre, il ne faut pas oublier qu'à l'époque de l'opération la demanderesse était déjà âgée de cinquante ans et avait deux enfants, c'est-à-dire qu'elle avait un âge où le sexe n'est pas aussi important que plus jeune, cette importance diminuant avec l'âge $»^{1}$. 
4 Les requêtes contre cette dernière décision ayant été rejetées, la requérante a attaqué le gouvernement portugais devant la $\mathrm{CEDH}$, lui reprochant d'avoir rendu une solution discriminatoire à raison tant de son âge que de son sexe.

5 À titre préliminaire, la CEDH rappelle que son rôle n'est pas de réévaluer le dommage, mais plutôt de dire si la décision d'appel au regard de sa motivation constitue une violation de l'article 14 de la Convention européenne des droits de l'homme relatif à la prohibition des discriminations et de l'article 8 de la Convention, qui proclame le droit au respect de la vie privée de la requérante.

6 À cet égard, la CEDH conclut à une violation desdits articles. Elle estime que les raisons pour lesquelles le montant octroyé au titre du préjudice moral est abaissé véhiculent un stéréotype entraînant une discrimination à l'encontre de la requérante, et donc une atteinte au respect de sa vie privée. Selon la Cour, la demanderesse subit un traitement particulier du fait de sa condition de femme âgée de cinquante ans, ce qui caractérise l'existence d'un traitement discriminatoire. En ce sens, l'importance de son épanouissement personnel à travers sa sexualité est niée, ce qui permet de caractériser une violation de son droit au respect de sa vie privée. Ainsi, la violation de l'article 8 de la Convention découle de celle de l'article14: c'est bien la discrimination qui est ici première. C'est la raison pour laquelle nous nous concentrerons sur cet élément.

7 Sur ce point, la spécificité de la décision rendue par la CEDH réside dans le fait qu'elle met en avant une conception particulière de la notion de discrimination qui, si elle n'est pas totalement absente de la jurisprudence antérieure de la Cour, n'est pas pour autant courante ${ }^{2}$.

8 La conception classique de la discrimination dans les arrêts de la CEDH résulte de la méthode dite "des trois étapes $»^{3}$, qu'il convient de rappeler brièvement. Selon cette méthode, pour pouvoir considérer qu'une mesure est discriminatoire il faut vérifier trois points: $1^{\circ}$ ) tout d'abord, il faut identifier deux situations identiques (ou du moins substantiellement analogues) et établir que les personnes placées dans ces situations peuvent être distinguées par un critère précis ${ }^{4} ; 2^{\circ}$ ) ensuite, il faut établir que la personne qui prétend être discriminée subit une différence de traitement en raison du critère en cause ; $3^{\circ}$ ) enfin, il convient de démontrer que cette différence de traitement n'est pas justifiée par une raison objective et raisonnable, c'est-à-dire qu'elle ne poursuit pas un but légitime ou qu'elle n'est pas proportionnée au regard du but recherché5. Dès lors que les trois étapes sont vérifiées, la CEDH condamne la discrimination, que celle-ci soit due à la rédaction d'un texte ou à son application.

9 Or, à cet égard, les faits de l'espèce n'étaient pas sans poser difficulté. Certes, la juridiction portugaise affirmait clairement que le montant de l'indemnisation était, pour partie au moins, déterminé par l'âge de la requérante, mais il n'était pas établi que d'autres personnes placées dans une situation identique aient statistiquement obtenu davantage.

10 En l'espèce, la difficulté est contournée par la CEDH qui fait usage d'une autre conception de la discrimination : elle se concentre sur le fait que la juridiction d'appel portugaise a usé d'un stéréotype pour apprécier le montant de l'indemnisation. Selon la Cour, ce seul fait est en soit une discrimination prohibée par l'article 14 de la Convention et une atteinte au droit au respect de la vie privée. 
11 La décision commentée met en avant les failles de l'analyse classique de la discrimination et les atouts de l'analyse dont fait usage la Cour en l'espèce (I). Nous montrerons toutefois que cette nouvelle analyse n'est pas exempte de critiques (II).

\section{$1 \%$ - Les atouts de l'analyse renouvelée de la discrimination}

Dans cette affaire, l'analyse classique de la discrimination est mise de côté par la Cour car elle ne permettait pas de sanctionner le gouvernement portugais (A). C'est une approche plus originale qui est mise en avant, et qui montre toute son efficacité dans la lutte contre l'usage de stéréotypes dans les décisions de justice (B).

\section{A/ - Une analyse classique insuffisante pour lutter contre les stéréotypes}

13 La présente affaire révèle l'insuffisance de l'analyse classique de la discrimination pour sanctionner les différences de traitement injustifiées tant dans les situations d'intersectionnalité (1) que dans le cas très particulier de la réparation du dommage corporel (2).

\section{i/ - Les limites de l'analyse classique dans des situations d'intersectionnalité}

Les discriminations dites "intersectionnelles $\|^{6}$ sont celles où la victime relève de plusieurs catégories à la fois sans qu'il soit possible de distinguer un seul critère sur la base duquel elle aurait été discriminée ${ }^{7}$. Dans la présente affaire, si l'on retient l'analyse classique, il existe un flou sur le point de savoir quel critère de discrimination doit être pris en compte pour vérifier l'existence d'une différence de traitement. La victime auraitelle fait l'objet d'une différence de traitement parce qu'elle est une femme ou parce qu'elle est âgée de cinquante ans au moment des faits? La difficulté est précisément qu'elle pourrait bien être discriminée en tant que « femme âgée ».

Si l'on considère la phrase litigieuse dans l'arrêt d'appel portugais, il semble que l'âge soit l'unique critère de fixation du montant de l'indemnisation. Il existe cependant une incertitude sur la question, la demanderesse - comme d'ailleurs la CEDH - se situant tantôt sur le terrain de l'âge, tantôt sur celui du sexe. Cette situation particulière de double critère d'identification possible conduit, dans l'analyse classique, à ce qu'il soit excessivement difficile d'identifier avec quelle autre personne la situation de la victime doit être comparée pour caractériser la différence de traitement. Ainsi, si l'on retenait le critère de l'âge, il faudrait comparer le traitement de la victime avec celui de personnes placées dans une situation similaire et ayant pour seule différence leur âge. Il conviendrait, par exemple, de comparer la situation de la victime avec celles de personnes de moins de cinquante ans. En revanche, si l'on retenait le seul critère du sexe, il faudrait comparer le traitement de la victime avec celui de personnes ayant pour seule différence leur sexe, en l'occurrence des hommes.

Mais aucune de ces deux options ne rend vraiment compte du problème spécifique de l'intersectionnalité. La juge Yudkivska, dans son opinion concordante, indique ainsi que, selon elle, si l'âge est bien le seul critère mis en avant par la juridiction portugaise, c'est 
une subtile combinaison des critères d'âge et de sexe qui serait ici opérée. Selon la juge, la victime est discriminée en raison d'une certaine perception de la sexualité féminine: parce qu'elle est une femme de cinquante ans, elle n'est probablement plus fertile, et donc plus aussi intéressée par la sexualité ; comme si les femmes n'avaient d'attrait pour la vie sexuelle que lorsque celle-ci est reproductive ${ }^{8}$. Dans cette situation ce n'est ni la seule qualité de femme qui était prise en compte par le juge portugais, ni la seule qualité de personne âgée mais bien la combinaison de ces deux critères qui se réalise dans une conception stéréotypée de la sexualité de la victime. On voit bien dans cette situation que le choix d'un seul critère de comparaison est impuissant à identifier le groupe de référence avec lequel la situation de la victime devrait être comparée.

L'analyse retenue en l'espèce par la CEDH, qui conduit à sanctionner la discrimination sur la base d'un stéréotype, permet en quelque sorte de prendre la différence de traitement «telle qu'elle est » (une femme âgée de cinquante ans, moins intéressée par les relations sexuelles que les femmes plus jeunes ou que les hommes du même âge, doit voir l'indemnisation de son préjudice minorée) sans avoir à poser la délicate question du critère en cause. Cette conception particulière de la discrimination permet en outre de pallier une seconde limite de l'analyse classique.

\section{ii/ - Les limites de l'analyse classique dans le domaine de la réparation du dommage corporel}

Quand bien même on choisirait abstraitement, dans cette affaire, de se concentrer sur le critère du sexe ou de l'âge, c'est-à-dire de mettre de côté le problème spécifique de l'intersectionnalité, l'analyse classique ne permettrait pas de prouver qu'il y a ici une différence de traitement.

19 Comme le soulignent les opinions divergentes, si la différence de traitement porte sur une question de droit, alors il suffit de montrer qu'une seule décision affirme un principe défavorable à une catégorie de personne pour démontrer la discrimination'. Mais lorsqu'il s'agit d'une question de fait, d'application concrète d'une norme à des cas particuliers, la preuve de la discrimination ne peut se faire qu'en montrant que, structurellement, un groupe se voit traiter moins favorablement qu'un autre. Il faudrait donc, dans ces cas, réaliser, selon le critère retenu, une étude statistique.

En l'espèce, la réparation du préjudice implique de faire une appréciation in concreto de la situation de la personne pour apprécier quelles ont été pour elle les conséquences du dommage. Il s'agit donc d'une question de fait. Or, la preuve statistique est extrêmement délicate en la matière : que doit-on en effet considérer comme étant deux situations analogues? L'obstacle central de la caractérisation de la discrimination est alors que les situations de deux victimes de dommages corporels ne peuvent jamais être parfaitement similaires.

21 La CEDH s'essaye malgré tout à la comparaison en rapprochant la décision portugaise attaquée de deux autres solutions rendues au Portugal en 2008 et 2014. S'attachant au critère du sexe, elle analyse la réparation des dommages corporels - impliquant une atteinte à la sphère sexuelle - de deux hommes âgés respectivement de 59 et 55 ans. Dans l'affaire de 2008, l'homme obtient environ 225000 euros pour son préjudice moral, tandis qu'en 2014 la victime se voit octroyer la somme de 100000 euros $^{10}$. La Cour évoque donc l'idée que, manifestement, la victime femme de cette affaire avait perçu une indemnisation moindre. 
Mais la comparaison est fragile. Tout d'abord en raison du peu de décisions étudiées ${ }^{11}$. Ensuite parce que les juridictions, et donc les juges qui se sont prononcés sur chaque cas, n'étaient pas les mêmes. Et enfin, dernier point majeur, parce qu'en droit portugais, l'indemnisation du préjudice moral constitue une enveloppe susceptible d'englober de nombreux préjudices. Autrement dit, comparer les sommes «attribuées au titre du préjudice moral» en droit portugais n'a que peu de sens, puisque si les situations sexuelles des victimes sont comparables, de nombreux autres éléments de leur vie et de leur personnalité sont pris en compte à ce titre. Il est donc impossible d'affirmer que si ces hommes ont reçu une indemnisation plus élevée, c'est parce qu'ils sont des hommes. La différence d'indemnisation entre les deux victimes masculines mentionnées par la Cour est d'ailleurs très importante et celle de l'homme le moins indemnisé (100 $000 €)$ est plus proche de celle de la demanderesse en l'espèce $(50000 €)$ que de celle de l'homme le plus indemnisé $(225000 €)$.

De ce dernier point de vue, on pourrait facilement considérer que le droit français, avec son cloisonnement des préjudices, serait un terrain plus propice à la comparaison statistique. L'analyse montre cependant que de nombreuses difficultés ne rendent pas cette étude plus révélatrice en droit français : subsistent notamment les questions de la gravité du dommage, de la différence d'appréciation entre juridictions, de l'influence des demandes formulées par les requérantes, etc ${ }^{12}$.

Cependant, dans cette affaire, la comparaison de la situation spécifique de la victime avec deux solutions particulières concernant des hommes fait apparaître un élément intéressant. Comme le relève la CEDH, les facultés reproductrices des deux hommes ne sont pas évoquées dans les décisions et leur âge n'est pas explicitement mis en relation avec leur préjudice sexuel. C'est donc moins la question quantitative de la réparation que la question qualitative de la motivation de la décision qui est en cause. Et c'est d'ailleurs sur ce point que la discrimination va être caractérisée par la Cour.

\section{B/ - Une analyse nouvelle propre à lutter contre les stéréotypes}

Dans cette décision, la violation de l'article 14 de la Convention n'est pas constituée par le biais des trois étapes précédemment exposées mais par le fait qu'à un moment donné, une considération stéréotypique est utilisée dans la motivation d'une décision de justice. La Cour précise cependant que la discrimination est liée au fait que ce stéréotype est utilisé dans un sens défavorable à la victime, puisque son indemnisation est réduite.

Cette approche de la discrimination est intéressante à double titre. Tout d'abord parce qu'elle contourne les difficultés statistiques que nous venons d'évoquer : elle permet de sanctionner des démarches discriminatoires pour lesquelles les victimes rencontrent d'énormes difficultés probatoires. Par ailleurs, au-delà de la situation particulière de la requérante, cette approche permet de sanctionner les juridictions qui, en utilisant les stéréotypes, participent à leur légitimation, voire à leur renforcement.

Dans son opinion concordante, la juge Yudkivska souligne ainsi que les juges portugais ont, dans cette affaire, failli dans leur rôle de remise en cause des stéréotypes sociaux et que cette attitude est en elle-même suffisante à caractériser une atteinte aux droits de la requérante, et notamment à sa dignité. Elle rappelle par-là que le droit n'a pas qu'une fonction régulatrice - par des décisions particulières - mais est aussi un outil d'affirmation de valeurs sociales ${ }^{13}$. Les décisions de justice, parfois malgré elles, ont en 
effet un impact social, médiatique, et peuvent être considérées comme porteuses d'une certaines " vérité judiciaire ». On pourrait donc souhaiter qu'elles soient irréprochables sur le plan des valeurs exprimées, au risque de faire douter de l'impartialité de la justice dans son ensemble.

Cette décision de la CEDH s'affirme donc comme exemplaire et inscrit d'ailleurs l'action de la Cour dans une politique plus globale de lutte contre les stéréotypes de genre menée au niveau international par le Conseil de l'Europe ${ }^{14}$. L'arrêt souligne ainsi que «l'égalité de genre est aujourd'hui un objectif majeur pour les membre du Conseil de l'Europe et par conséquent des motifs très sérieux doivent être mis en avant pour qu'une différence de traitement (entre homme et femme) soit considérée comme compatible avec la Convention $»^{15}$.

Pour autant, si l'objectif semble louable - et peut dans de nombreuses situations être tout à fait efficace - la méthode n'est pas sans prêter le flanc à la critique.

\section{$2^{\circ} /$ - Les limites de l'analyse renouvelée de la discrimination}

Si l'on suit jusqu'au bout le raisonnement de la Cour, on doit considérer que l'utilisation par une juridiction, d'un stéréotype pour motiver sa décision est par principe discriminatoire. Mais cette position pose de nombreuses difficultés (A) qui ne trouvent que des solutions insatisfaisantes (B).

\section{A/ - Les difficultés multiples}

31 Trois limites à l'analyse de la discrimination en termes de stéréotypes peuvent être observées. Tout d'abord la question de l'appréciation négative portée sur la situation de la victime (1), ensuite la difficulté même d'identifier les stéréotypes dans les décisions étudiées (2), et enfin les effets pervers qui pourraient se faire jour dans le fait d'écarter totalement une réflexion stéréotypique (3).

\section{i/ - La place de l'appréciation négative portée sur la victime}

La lecture de la décision de la CEDH suscite immédiatement une question théorique : cette «nouvelle » conception de la discrimination suppose-t-elle toujours que l'on prouve que le stéréotype a influencé de façon négative la situation de la personne?

L'hypothèse est théorique parce qu'il est peu probable que quelqu'un engage une procédure judiciaire pour avoir été traité de façon favorable par rapport à un tiers. Mais la question n'en est pas moins susceptible de se poser, ne serait-ce que dans une dimension militante ${ }^{16}$. En l'espèce la Cour note que l'indemnisation a été diminuée. Or, si le traitement négatif est toujours nécessaire à la caractérisation de la discrimination, alors on manque l'objectif politique pourtant souligné par la Cour. À l'inverse, si l'on considère que le traitement négatif n'est pas indispensable, alors on pourrait s'interroger sur le point de savoir si tout un chacun n'aurait pas intérêt à agir contre une décision stéréotypée, dès lors que c'est l'image de la justice qui est en jeu, davantage qu'une situation individuelle. Mais cette interrogation suppose, en amont, de considérer que 
l'identification d'un stéréotype est une démarche évidente. Cela n'est pourtant pas si flagrant.

\section{ii/ - La difficulté pratique d'identifier une affirmation stéréotypée}

La question de savoir à partir de quand une motivation peut être caractérisée comme stéréotypée n'est pas si simple qu'il y parait au premier abord. D’ailleurs, cela ne l'était pas dans la présente affaire. Reprenons la phrase litigieuse : on nous dit que l'importance accordée à la sexualité diminue avec l'âge. Si l'on considère que cette affirmation s'applique à la victime elle-même alors il s'agit bien d'un motif stéréotypé, potentiellement faux. Mais ce n'est là qu'une interprétation possible de la motivation observée. Il était en effet envisageable de lire la décision comme affirmant que l'importance du sexe dans l'évaluation du préjudice moral diminue avec l'âge. Or, cette affirmation est moins contestable puisque, l'âge avançant, les victimes supporteront moins longtemps leur préjudice. Selon cette interprétation, « l'importance du sexe » dans l'évaluation du préjudice moral diminue bel et bien avec l'âge: il ne s'agit pas d'un stéréotype mais d'une définition de la notion. Il n'est d'ailleurs pas discuté que l'âge soit pris en compte dans l'indemnisation du préjudice corporel et la CEDH rappelle d'ailleurs clairement que « le problème n'est pas la considération de l'âge ou du sexe en tant que tels, mais plutôt la supposition selon laquelle la sexualité n'est pas aussi importante pour une femme de 50 ans mère de deux enfants que pour une personne plus jeune $»^{17}$.

Identifier la formulation d'un stéréotype n'est donc pas si évident et, dans cette affaire, la CEDH prend d'ailleurs soin de justifier son interprétation. D'abord en contextualisant la phrase litigieuse. La Cour relève à cet égard que sur une autre question, celle de l'évaluation du préjudice matériel, la juridiction portugaise avait minoré la somme attribuée à l'embauche de personnel de service en arguant de ce que, les enfants de la victime étant âgés, celle-ci "n'aura besoin que de prendre soin de son mari ${ }^{18}$. L'interprétation de la CEDH semble par ailleurs influencée par plusieurs rapports dénonçant les préjugés à l'œuvre dans la justice portugaise ${ }^{19}$. En somme, en l'absence d'éléments de contexte, des difficultés peuvent apparaître quant à la caractérisation même du stéréotype, qui ne saurait être sanctionné que s'il est clairement exprimé $^{20}$. Une autre difficulté est celle de l'usage de stéréotypes dont la victime ressentirait des réels effets.

\section{iii/ - La place des stéréotypes vécus}

Le caractère ambivalent du stéréotype réside dans le fait qu'il est parfois construit à partir d'une généralisation. La difficulté qui peut alors se présenter si l'on rejette absolument tout motif stéréotypique est que l'on risque de priver les juges d'arguments qui pourraient être favorables à la victime. En effet, le stéréotype, s'il peut être faux pour une personne considérée peut également avoir pour elle une certaine réalité. Par exemple, l'affirmation générale selon laquelle «l'apparence est importante pour les femmes ", pourrait conduire à réparer plus généreusement le préjudice esthétique subi par une femme en particulier. Or, si cette affirmation est sans doute fausse pour une partie des femmes comme des hommes, il reste vrai que les femmes subissent socialement davantage de jugements portant sur leur apparence physique ${ }^{21}$. La victime n'en a pas forcément conscience, ne se l'exprime pas nécessairement, peut difficilement le prouver, mais de fait son préjudice sera plus grand. Dès lors, priver les juridictions de cette facilité 
de motivation n'est-ce pas vouloir procéder à une universalisation de la parole judiciaire qui, de fait, jouerait contre les victimes placées par ailleurs, du fait de leur position sociale, dans des positions stigmatisées? Une solution possible à cet écueil, serait de s'appuyer uniquement sur la parole de la victime.

\section{B/ - Des solutions insatisfaisantes}

Une piste de réflexion permettant de distinguer les usages légitimes et illégitimes des stéréotypes serait de considérer que la « réalité » des effets du stéréotype ne peut être définie que par la victime elle-même (1). Cette solution n'est cependant pas sans susciter des difficultés pratiques (2).

\section{i/ - La possible prise en compte de la parole de la victime}

Dans le champ particulier du préjudice sexuel, il serait possible de considérer que l'appréciation la plus juste du préjudice ne peut être le fait que de la victime elle-même. Elle est en effet la seule en capacité de retranscrire fidèlement l'état de sa vie sexuelle, au regard de ses propres critères de ce qu'est une vie sexuelle «normale ».

Dans cette affaire, la CEDH semble d'ailleurs aller dans ce sens en rattachant la question de la discrimination à celle de la vie privée, ici entendu comme le droit au libre épanouissement de sa personnalitée 22 . On peut ainsi penser que la dimension sexuelle de l'existence étant une question profondément identitaire, la « vérité » de cette notion ne saurait être détenue que par la personne considérée.

Concrètement, cela reviendrait à considérer que si la victime motive sa demande d'indemnisation par un stéréotype, alors celui-ci devrait être pris en compte. Ainsi des affirmations telles que « je suis un homme, j'ai subi une ablation de la verge ce qui me cause un préjudice particulièrement important dans la mesure où cet organe est socialement considéré comme étant au cœur de mon identité masculine » ou « je suis une femme et le retrait de mon utérus heurte profondément mon identité féminine" pourraient être prises comme reflétant une certaine réalité du préjudice subi, malgré leur aspect stéréotypé. L'idée serait alors de reconnaître qu'en se sentant « moins homme » ou "moins femme", les personnes qui ont adhéré à ces stéréotypes sociaux et les ont intégrés pour construire leur identité subissent un préjudice réel.

41 En revanche, si le stéréotype était "projeté » sur la personne par la juridiction ou la partie adverse, alors il devrait être considéré comme discriminatoire. C'est le cas en l'espèce puisqu'est imposée à la victime l'affirmation selon laquelle elle est une femme de cinquante ans ayant déjà deux enfants et que pour cette raison la vie sexuelle a moins d'importance pour elle que pour une personne plus jeune. Bien sûr une telle position ne serait pas sans écueils.

\section{ii/ - Les difficultés pratiques de la prise en compte de la parole de la victime}

S'appuyer uniquement sur la parole de la victime pour évaluer son préjudice sexuel se heurte à deux difficultés majeures.

D'une part, les victimes pourraient être tentées d'adapter leur discours en faisant leurs des affirmations stéréotypées dans l'espoir d'augmenter leur indemnisation, sous 
l'influence notamment de conseils aguerris. On peut toutefois se demander si cela n'est pas d'ores et déjà une pratique à l'œuvre.

D'autre part, il est possible qu'un grand nombre de considérations sociales (statut social, aspects culturels et ethniques, âge, genre ${ }^{23}$ etc.) puissent conduire certaines victimes à éprouver plus de difficultés que d'autres à évoquer et décrire spontanément leurs difficultés d'ordre sexuel. Or si une telle hypothèse était vérifiée, elle constituerait un obstacle au fait de s'en remettre à la seule parole de la victime pour déterminer l'importance de préjudice, au risque d'accentuer les inégalités que l'on souhaite voir disparaître par la lutte contre les stéréotypes ${ }^{24}$. Le combat est donc bien plus complexe que cette décision ne pourrait le faire croire.

Lutter contre l'usage de stéréotypes dans les motivations des juridictions, comme le fait ici la CEDH, est sans nul doute une démarche nécessaire. Cela permet à l'évidence d'éviter que ces énoncés normatifs ne véhiculent des messages porteurs de préjugés et de stigmatisations. Cette position permet en outre de rappeler aux juridictions la nécessité d'une appréciation réellement individuelle des situations, notamment dans la réparation du dommage corporel.

Cependant, il convient de ne pas faire preuve ici de naïveté : on sait que le langage s'adapte plus vite que les esprits. Une justice qui n'exprime pas explicitement les stéréotypes qui la traversent n'en est pas pour autant exempte. L'application du droit peut demeurer profondément discriminatoire même lorsque son discours s'est, en apparence, neutralisée ${ }^{25}$. La recherche ne doit pas renoncer devant les difficultés statistiques, probatoires etc. mais continuer d'user d'une lecture critique du droit pour en faire évoluer les présupposés, pour profondément renouveler le regard posé sur les personnes, leurs identités, leurs relations, leurs sexualités, au-delà des mots.

CEDH, 25 juill. 2017, Carvalho Pinto De Sousa Morais c. Portugal, $n^{\circ}$ 17484/15

Les Lettres " Actualités Droits-Libertés» (ADL) du CREDOF (pour s'y abonner) sont accessibles sur le site de la Revue des Droits de l'Homme (RevDH) - Contact

\section{NOTES}

1. Nous traduisons en français à partir de la version anglaise de la décision de la CEDH, v. CEDH 25 juill. 2017, n 17484/15, aff. Carvalho Pinto De Sousa Morais c. Portugal, § 16.

2. V. not. CEDH 24 juin 1993, $n^{\circ}$ 14518/89, aff. Schuler-Zraggen c. Suisse, § 67 ; CEDH 25 mai 2010, n - 37193/07, aff. Paraskeva Tororova c. Bulgarie, § 38-40; CEDH, 20 mai 2010, n 38832/06, aff. Alajos Kiss v. Hungary, $\S 42$; CEDH, grande Chambre, 22 mars 2012, n 30078/06, aff. Konstantin Markin c. 
Russie, $\$ 141$; contra CEDH, grande chambre, 27 juillet 2010, $n^{\circ}{ }^{s} 4149 / 04$ et 41029/04, aff. Aksu v. Turkey, § 45.

3. V. l'opinion divergente des juges RAVARANi et BOSNJAK, $n^{\circ} 7$ et $\mathrm{s}$. En général, sur le mécanisme de qualification de la discrimination v. Fr. SUDRE, Droit européen et international des droits de l'Homme, $13^{e}$ éd., PUF, 2016, $n^{\circ} 278$ et s. Plus spécialement en droit européen v. Fr. SUDRE et alii, Les grands arrêt de la Cour européenne des droits de l'Homme, $8^{\mathrm{e}}$ éd., PUF, 2017, p. 101 et s.

4. L'article 14 de la Convention européenne des droits de l'hommes vise le sexe, la race, la couleur, la langue, la religion, les opinions politiques ou toutes autres opinions, l'origine nationale ou sociale, l'appartenance à une minorité nationale, la fortune, la naissance ou toute autre situation.

5. Ex. CEDH 28 mai 1985, $\mathrm{n}^{\circ}{ }^{\mathrm{s}} 9214 / 80,9473 / 81$ et 9747/81, aff. Abdulaziz, Cabales et Balkandali c. Royaume-Uni, § 72 ; CEDH 13 déc. 2011, n³1827/02, aff. Laduna c. Slovaquie, § 59 ; CEDH 25 juill. 2017, préc., § 44 .

6. Certains auteurs distinguent nettement les deux expressions en considérant que "l'intersectionnalité constitue plus qu'une simple analyse des discriminations multicritères (encore parfois appelée "discriminations multiples") constatées lorsque plusieurs critères de discrimination se mêlent sans lien immédiat. Par exemple, une femme en fauteuil roulant peut à la fois se trouver confrontée, professionnellement, au plafond de verre et, dans sa vie quotidienne, à des difficultés de déplacement et d'accès à certains immeubles. Dans ce cas de figure, les obstacles et les expériences de discrimination à raison du sexe et du handicap se cumulent, sans toutefois être nécessairement liés. L'intersectionnalité désigne, en revanche, les situations dans lesquelles il est impossible de distinguer les fondements à l'origine de la discrimination (ni même celui qui joue la plus grande part dans la discrimination) ", S. HENNETTEVAUCHEZ, M. PICHARD et D. ROMAN (dir.), La loi \& le genre - Études critiques de droit français, CNRS éditions, Paris, 2014, p. 693. À notre sens, la frontière entre les deux n'est pas si nette: la discrimination intersectionnelle apparaît comme une sorte de discrimination multiple.

7. M. MöSCHEL, «L'intersectionnalité dans le contentieux de la non-discrimination relatif au domaine de l'emploi en France ", in S. HenNETTE-VAuchez, M. PichaRd et D. RomAn (dir.), La loi \& le genre, préc., p. 697 ; J. Roux, «L'égalité entre (toutes) les femmes et les hommes - Les mutations $\mathrm{du}$ droit vers la protection contre les discriminations multiples et intersectionnelles ", La Revue des droits de l'Homme [en ligne], 7 | 2015, mis en ligne le 22 mai 2015, consulté le 11 août 2015 [http://revdh.revues.org/1116]. De manière générale v. S. BILGE et P. HILL COLLINS, Intersectionality, New-York, Polity, 2016.

8. Opinion concordante de la juge Yudkivska sous CEDH 25 juill. 2017, préc., p. 22.

9. On peut penser notamment à la décision $\mathrm{CEDH}$, Grande chambre, 22 janvier 2008, aff. E. B. c. France, $\mathrm{n}^{\circ}$ 43546/02.

10. L'âge est évoqué dans la première décision, pas dans la deuxième. Dans aucune des deux on trouve de référence aux facultés procréatrices des hommes.

11. Cela est d'ailleurs relevé dans les opinions dissidentes des juges RAVARANI et BOSNJAK (p. 37, § 25).

12. Pour une analyse plus approfondie, v. L. CARAYon, M. DuguÉ, J. MATtiusSI, « Réflexions autour du préjudice sexuel », D. 2017. 2257.

13. V. par ex., parmi une littérature abondante, M. MIALLE, Une introduction critique au droit, éd. François Maspero, $2^{\mathrm{e}}$ éd., 1982, not. p. 108-109 : « le système juridique est devenu, parmi tous les systèmes normatifs, celui qui a conquis l'hégémonie dans la fonction de "dire" la "valeur des actes sociaux". [...] La morale ou la religion ayant été reléguées au rang de prise de position individuelle, le droit paraît être le seul système objectif de qualification des rapports sociaux : il est donc d'autant plus valorisé dans cette fonction. Il est même identifié à ce qu'il réalise, c'est-àdire à la valeur de ces rapports ». 
14. «Combattre les stéréotypes de genre et le sexisme» est ainsi le premier objectif de la Stratégie du Conseil de l'Europe pour l'égalité entre les femmes et les hommes 2014-2017, p. 9 et s.

15. CEDH, 25 juill. 2017, préc., §46. Cet objectif était déjà invoqué comme justification à la discrimination par stéréotype dans CEDH, 24 juin 1993, n 14518/89, aff. Schuler-Zraggen c. Suisse, $\S 67$.

16. Ainsi, l'affirmation selon laquelle l'indemnisation d'un préjudice esthétique devrait être augmentée pour une requérante au motif que l'apparence est nécessairement importante pour une belle jeune femme, susciterait sans doute l'indignation d'une demanderesse féministe...

17. CEDH 25 juill. 2017, préc., 552.

18. Ibid., § 54 .

19. V. not. Rapport de la Rapporteuse spéciale sur l'indépendance des juges et des avocats, Gabriela KNAUL - mission au Portugal, § 72, observations finales concernant les huitième et neuvième rapports périodiques du Portugal, Comité pour l'élimination de la discrimination à l'égard des femmes, 24 novembre 2015, $\$ 20$ et 21 (sur les stéréotypes au Portugal en général), cités par CEDH 25 juill. 2017, préc., \$ 26, 28 ; V. aussi le rapport de l’observatoire permanent de la justice portugaise de novembre 2016, disponible en portugais et cité par CEDH 25 juill. 2017, préc., § 29.

20. Sur ce point v. L. CARAYon, M. Dugué, J. Mattiussi, «Réflexions autour du préjudice sexuel », art. préc.

21. V. par ex. D. LE BRETON, «D'une tyrannie de l'apparence : corps de femmes sous contrôle », in Éthique de la mode féminine, PUF, 2010, p. 208. Pour un exemple précis de l'apparence pour des femmes en milieu professionnel masculin v. J. THOMAs, «Le corps des filles à l'épreuve des filières scolaires masculines ", Sociétés contemporaines, 2013/2 ( $\left.n^{\circ} 90\right)$, p. 122.

22. CEDH, rapport du 12 juill. 1977, $n^{\circ}$ 6959/75, aff. Brüggemann et Scheuten c/ RFA \$54: DR 10, p. 100, spéc. p. 137 ; CEDH 6 févr. 2001, n 44599/98, aff. Bensaid c. Royaume-Uni, §47 : «L'article 8 protège un droit à l'identité et à l'épanouissement personnel et celui de nouer et de développer des relations avec ses semblables et le monde extérieur »; CEDH 14 mai 2002, n 38621/97, aff. Zehnalova et Zehnal c. Rép. Tchèque : «droit [au] développement personnel et [...] droit d'établir et d'entretenir des rapports avec d'autres êtres humains et le monde extérieur »; CEDH 8 janv. 2009, n² 29002/06, aff. Schlumpf c. Suisse, \$77 : « Le droit au respect de la vie privée inclut le droit au développement personnel et le droit d'établir et d'entretenir des rapports avec les autres êtres humains et le monde extérieur »; CEDH 27 mai 2014, n 10764/09, aff. de la Flor Cabrera c. Espagne, $\S 30$ : « la Cour rappelle que la notion de "vie privée" est une notion large, non susceptible d'une définition exhaustive, qui recouvre l'intégrité physique et morale de la personne et peut donc englober de multiples aspects de l'identité d'un individu, tels le nom ou des éléments se rapportant au droit à l'image »; F. SUDRE, «Rapport introductif. La "construction" par le juge européen du droit au respect de la vie privée », in F. SUDRE (dir.), Le droit au respect de la vie privée au sens de la Convention européenne des droits de l'Homme, Droit et justice 63, Bruylant, Bruxelles, 2005, p. 11 ; v. aussi S.-M. FERRIÉ, Le droit à l'autodétermination de la personne humaine - Essai en faveur du renouvellement des pouvoirs de la personne sur son corps, th. dactyl. Paris 1, 2015, $\mathrm{n}^{\circ} 160$ et s., p. 111 et s.

23. Pour la question du genre, v. L. CARAYON, M. Dugué, J. MATTIUSSI, art. préc.

24. D'autres solutions, conduisant à réformer profondément notre système de réparation du dommage corporel pourraient ainsi être explorées : forfaits d'indemnisation, revalorisation du rôle de l'assurance-maladie dans la prise en charge des personnes victimes de dommage corporels etc. V. par ex. Ph. LE TOURNEAU (dir.), Droit de la responsabilité et des contrats, Régimes d'indemnisation, $10^{\mathrm{e}}$ éd., Dalloz action 2014-2015, n 91 ; L. MÉLENNEC, L'indemnisation du handicap, 
Pour l'instauration d'un régime unique de l'invalidité et de la dépendance, Desclée de Brouwer, 1997, spéc. p. 163 et s.

25. Sur la question du genre, pour un aperçu général, v. not., S. HenNETTE-VAUCHEZ, M. PiCHARD et D. Roman (dir.), La loi \& le genre - Études critiques de droit français, CNRS éditions, Paris, 2014. Pour une réflexion sur cette question au regard de la race dans le système juridique américain $\mathrm{v}$. l'ouvrage majeur, récemment traduit en français: M. ALEXANDER La couleur de la justice. Incarcération de masse et nouvelle ségrégation raciale aux États-Unis, trad. Anika Scherrer, éd. Syllepse, 2010, 2017 pour la version française.

\section{RÉSUMÉS}

Le 25 juillet dernier, la Cour européenne des droits de l'homme (CEDH) a rendu une décision dans laquelle elle estime qu'il est discriminatoire de réduire l'indemnisation du préjudice moral d'une personne ayant subi un dommage corporel atteignant les organes sexuels au motif qu'il s'agit d'une femme âgée de cinquante ans.

\section{AUTEURS}

\section{LISA CARAYON}

Maitresse de conférences à l'Université Paris 13 - IRIS

\section{JULIE MATTIUSSI}

Docteure en droit, enseignante-chercheuse à l'Université de Cergy-Pontoise 